Background and Aims Primary outcome was to assess if femoral nerve block (FNB) could affect long-term pain and function after total knee arthroplasty (TKA) (1).

Methods Ethics Committee approved this retrospective study, patients (age 18-90 years) operated from unilateral TKA one year before were included and divided in 2 groups: patients receiving spinal anesthesia (hyperbaric bupivacaine \pm sufentanil) or spinal anesthesia with FNB (50-100 mg levobupivacaine). Impact of age, gender, BMI, height, durations of surgery and tourniquet over the pain and disability were also evaluated. Patients were asked to evaluate pain before (NRS) and after TKA with the Numeric Rating Scale (NRS1) and functional disability with the Oxford Knee Score one year after surgery (OKS1).

Results Continous variables were compared between groups with Wilcoxon test, discrete variables with $\mathrm{Chi}^{2}$ Pearson test; $\mathrm{p}<0.05$ significant. 111 patients were included (24 with FNB) There is no difference in term of OKS1 (42 [39 - 46] vs 43.5 [38.75 - 46]; p=0.87) when FNB is added to spinal anesthesia. Median OKS1 is superior for men compared to women (41 [37.75 - 44.25] vs 45 [40 - 46]; $\mathrm{p}=0.01)$. When comparing OKS $1>40$ with $\mathrm{OKS} 1<40$, in group OKS1>40: Median Height and delta (NRS1-NRS) are superior (height: $1.65[1.56-1.71]$ vs 1.68 [1.63 1.76]; $\mathrm{p}=0.02$. Delta NRS: $-4[-6$ - -2] vs $-6[-8-4]$ $\mathrm{p}<0.01)$; NRS1 is inferior $(4[2-5]$ vs $1[0-2]$; $\mathrm{p}<0.001)$.

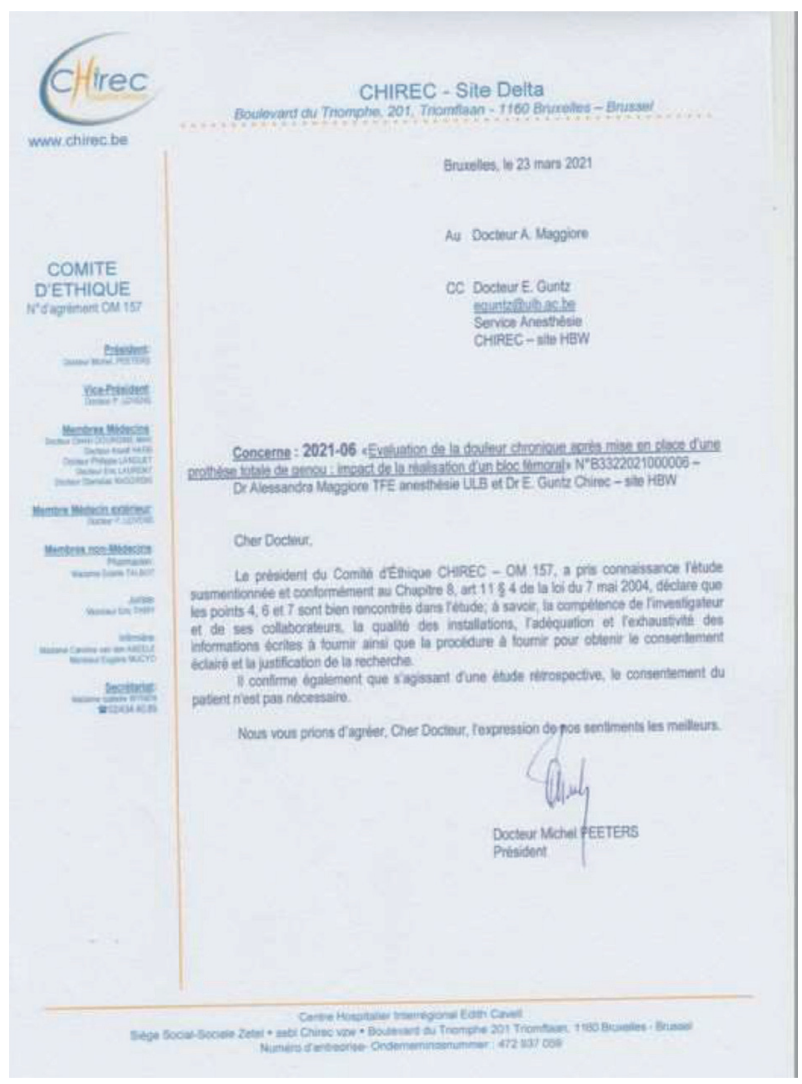

Abstract 144 Figure 1

Conclusions FNB does not improve long-term pain and knee function. Knee function seems to be improved by the decrease of pain, the gender and the height.

\section{A NOVEL CONVENIENT WAY OF PERFORMING MEDIAL APPROACH TO SCIATIC NERVE BLOCK IN SUPINE POSITION}

JWL Lim, Y Cheng. Ng Teng Fong General Hospital, Singapore, Singapore

\subsection{6/rapm-2021-ESRA.145}

Background and Aims Current approaches to sciatic nerve block either require position change, which is time consuming, or involves blocking the sciatic nerve deeper in the tissue. To perform the block in supine position, patient's knee is flexed in unsteady position, requiring assistant or sandbag under the ankle to maintain this position. Medial approach described in current literature position patient's leg in figure-of-four which is difficult in patients with hip or knee conditions. We propose a novel convenient way of positioning patient's lower limb of interest above contralateral ankle for medial approach to sciatic nerve block in two case studies.

Methods Sciatic nerve block was performed in two patients under ultrasound guidance with their lower limb of interest positioned above contralateral ankle.

Results Sciatic nerve block was performed successfully for anaesthesia intraoperatively in both patients. Our proposed position was time efficient with ease of positioning in supine position without need for support and femoral/saphenous nerve block was performed in the same position. Sciatic nerve appears more elongated on ultrasound when approached

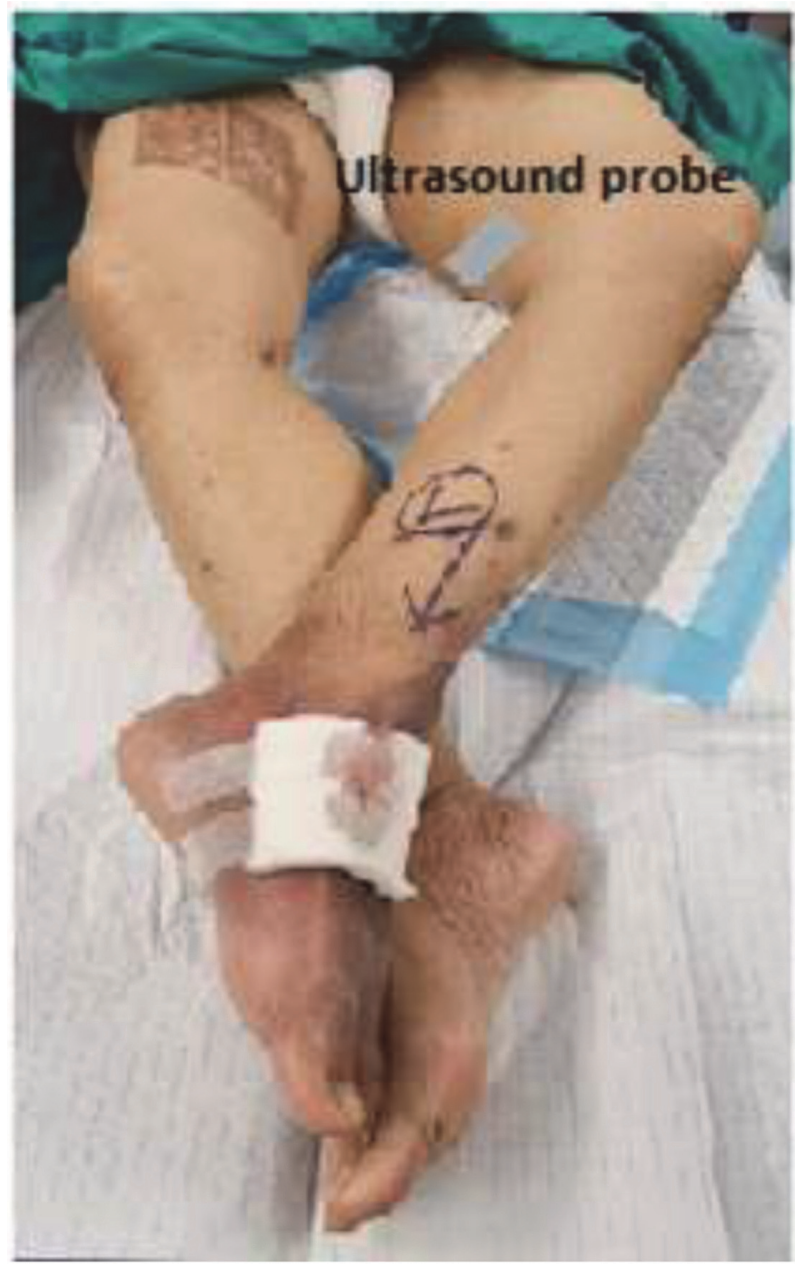

Abstract 145 Figure 1 


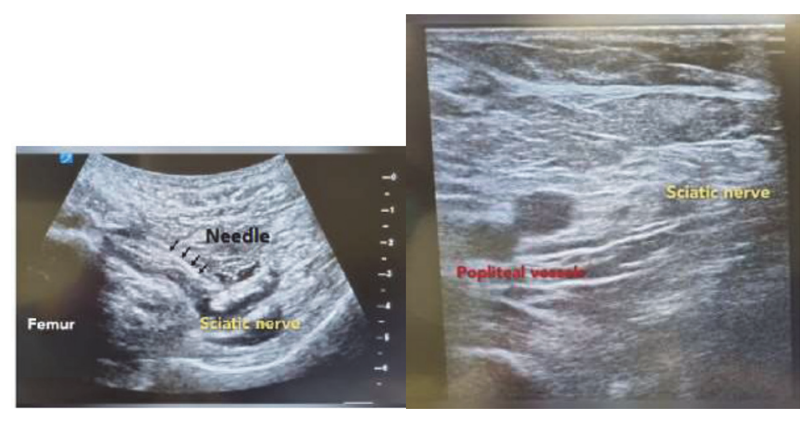

Abstract 145 Figure 2

medially, allowing greater surface area for spread of local anesthesia. Vascular structures were further from sciatic nerve, reducing the risk of intravascular injection of local anaesthesia. With tibial nerve approached first medially, risk of common peroneal nerve injury could be reduced compared to lateral approach.

Conclusions Our proposed position for medial approach of sciatic nerve block saves positioning time, with greater surface area for spread of local anesthesia, likely reducing the risk of intravascular injection of local anaesthesia and common peroneal nerve injury.

\section{ADDUCTOR CANAL BLOCK AND FEMORAL TRIANGLE BLOCK: COMPARISON OF TIME TO ACHIEVE DISCHARGE CRITERIA AND EVALUATION OF LOCAL ANESTHETIC SPREAD}

N Sakai* , C Taruishi, T Sudani. Daiyukai General Hospital, IChinomiya, Japan

\subsection{6/rapm-2021-ESRA. 146}

Background and Aims The femoral triangle block (FTB) and adductor canal block (ACB) have become standard analgesia for total knee arthroplasty (TKA). We compared the anatomical difference and postoperative recovery between two blocks. Methods We randomly assigned 118 patients to the FTB or ACB group. Patients were given $10 \mathrm{~mL}$ of $0.25 \%$ levobupivacaine as FTB or ACB. FTB was defined as at the mid-thigh, and $\mathrm{ACB}$ at the apex of the femoral triangle. The primary outcome was to achieve the discharge criteria (pain control with oral analgesics, knee flexion $>90^{\circ}$, and ambulatory rehabilitation). ACB would be noninferior to FTB if the $95 \%$ confidence interval of the two groups' differences were closer to zero than -9 hours (margin). We compared the local anesthetic spread, straight leg raise (SLR), and other outcomes.

Results The time to achieve discharge criteria was $56.3 \pm 17.3$ hours in the ACB group and $56.2 \pm 18.4$ hours in the FTB group, a difference of 0.1 hours (95\% CI: -6.4-6.6 hours, $\mathrm{p}=0.97$ ), establishing noninferiority. At one hour postoperatively, 48 of 60 patients in $\mathrm{ACB}$ and 40 of 58 patients in FTB were capable of SLR (Odds ratio:0.59, p=0.29), a nonsignificant difference. The distance between the two points was $5.1(4.9-5.4) \mathrm{cm}$, and the spread of local anesthetics was $6.9 \mathrm{~cm}$ cephalad, $5.1 \mathrm{~cm}$ caudad. There were no differences in pain scores or other outcomes.

Conclusions ACB was non-inferior to FTB in time to achieve discharge criteria. We must warn of the potential quadriceps weakness after local anesthetic injection because of the high cephalad spread.

\section{ERECTOR ERECTOR SPINAE BLOCK FOR ANALGESIA IN A PATIENT HAVING UNDERGONE CLAM SHELL THORACOTOMYSPINAE BLOCK FOR ANALGESIA IN A PATIENT HAVING UNDERGONE CLAM SHELL THORACOTOMY}

'S Park*, ${ }^{2}$ A Mcdaniel. 'Imperial College Healthcare NHS Trust, London, UK; ${ }^{2}$ St Mary's Hospital, London, UK

\subsection{6/rapm-2021-ESRA. 147}

Background and Aims A 16 year old male suffered a stab wound to right posterior lower thorax. CT showed a right hydrohaemopneumothorax \& right renal laceration and chest drain was inserted. The patient became unstable during opertation requiring a clam shell thoracotomy. The patient's haemodynamics improved with multiple blood product transfusions and repeat CT showed an enlarging right perinephric haematoma, and shattered right kidney. He was admitted to ICU for Respiratory weaning but adequate analgesia was challenging, coagulopathy and rising inflammatory markers ruled out epidural. Bilateral erector spinae plane infusions were sited to optimise analgesia.

Methods ESP catheters were inserted at T5 bilaterally. Nerve catheters were placed under ultrasound guidance and $60 \mathrm{ml}$ of $\quad 0.25 \%$ Levobupivacaine (adrenaline $1: 400 \quad 000$ ) loading dose administered. A continuous infusion of levobupivacaine at $15 \mathrm{ml} / \mathrm{hr}$ was commenced and bolus doses prescribed.

Results ESP catheter infusion continued at $10-15 \mathrm{ml} / \mathrm{hr}$ for 10 days with $40 \mathrm{ml}$ of $0.125 \%$ levobupivacaine bolus top-ups. The patient was extubated D10 ESP infusion and the infusion was weaned - patient reported 0/10 pain. He was transitioned to regular analgesics with regular pregabalin and PRN oxycodone \& discharged to the ward.

Conclusions ESP can be considered as a part of analgesia regime in appropriately selected patient groups. Multiple contra-indications exist in trauma settings. This patient's coagulopathy contraindicated epidural insertion. However, an ESP could be safely inserted, achieving a good level of analgesia.

Overall, the ESP played a pivotal role in this patient's ITU care and the overall recovery from his polytrauma.

\section{REVIEWING THE CURRENT REGIONAL ANAESTHESIA SERVICE FOR HIP FRACTURES AT A MAJOR TRAUMA CENTRE}

R Kulec*, A Hassan. Nottingham University Hospitals NHS Trust, Nottingham, UK

\subsection{6/rapm-2021-ESRA.148}

Background and Aims A Fractured neck of Femur can cause severe pain. There is overwhelming evidence that peripheral nerve blocks are effective in providing analgesia for this injury and reduce serious complications such as the risk of acute confusion, chest infection and time to first mobilisation. ${ }^{1}$

In 2018 a landmark Fascia Iliaca Block (FIB) service was set up at the Queen's Medical Centre in Nottingham. Since then, there have been new national recommendations supporting the use of ultrasound to provide a more accurate deposition of local anaesthetic and superior block. ${ }^{2,3}$

Methods Retrospective data collection on 101 patients who attended the emergency department with a neck of femur fracture. Their notes were reviewed electronically for information on demographics, FIB delivery, pain scores and opioid 